\title{
Deep-Space Optical Communications Link Availability and Data Volume
}

\author{
A. Biswas ${ }^{1}$ and S. Piazzolla ${ }^{1}$
}

\begin{abstract}
Optical links from a spacecraft at planetary distances to a ground-based receiver presume a cloud-free line of sight (CFLOS). If a network of ground-based optical receivers is used for providing a data service from deep space, site diversity of cloud cover will be exploited in order to improve availability. In this article, a preliminary estimate of achievable data volumes, downlinked from Mars distances to a 5-mdiameter telescope similar to the Hale telescope, is undertaken. The data volume is affected by the CLFOS duration and the variation of atmospheric parameters during the CFLOS. Simplified analysis using a cluster of ground stations to comprise each node of the global network provides conservative estimates of the data volumes, to range from 60 to 90 Gbits/day when Mars is at maximum range and the SunEarth-probe angles are 3 to $10 \mathrm{deg}$. On the other hand, when the spacecraft range is closer, the data volumes, constrained by the maximum data rate that the Mars Laser Communications Demonstration Project can support, will be approximately 450 Gbits/day.
\end{abstract}

\section{Introduction}

The Mars Laser Communications Demonstration (MLCD) Project [1-3] initiated by NASA has stimulated renewed interest in deep-space optical communications. In this article, link availability and data volumes from Mars to a ground-based optical network are discussed. MLCD link characteristics are used in presenting the discussion.

An Earth optical receiving network for providing data service to NASA's planetary spacecraft will be the subject of much future study. The available options are space, high-altitude platforms (for example, balloons), or ground. Space-based receiving stations overcome cloud-cover outages and performance degradation related to atmospheric perturbations of the downlink laser signal. Likewise, deploying receivers on balloons or other high-altitude platforms completely eliminates cloud blockage and largely mitigates atmospheric effects. Both space-based and high-altitude platforms will require special considerations for stabilizing the optical receiver line of sight. The design or cost to achieve this has not been addressed in any depth. On the other hand, a ground-based network with performance limited by availability and atmosphere-induced degradation is worth considering, from a cost standpoint, at least in the near term. For a ground-based network, the quality and continuity of data service affected by cloud

\footnotetext{
${ }^{1}$ Communications Architectures and Research Section.

The research described in this publication was carried out by the Jet Propulsion Laboratory, California Institute of Technology, under a contract with the National Aeronautics and Space Administration.
} 
blockage and atmospheric degradation need to be quantified in order to compare the performance penalty with the perceived cost saving. This article presents some preliminary discussions addressing this critical issue.

A future optical ground-based network would be comprised of nodes carefully selected for the prevalence of clear, cloud-free skies as well as low atmospheric attenuation, sky brightness, and good atmospheric "seeing." Cloud-cover statistics for selected nodes of the network can be utilized to determine an overall cloud-free line-of-sight (CFLOS) availability. During CFLOS, the large variability of the atmospheric parameters will influence link performance and, therefore, data volumes. Conceptually, the most effective ground networks not only will provide the maximum CFLOS but also will be comprised of nodes that provide a high percentage of relatively benign atmospheric conditions. Additionally, ground-station receiver design will be directed toward maintaining optical links at low Sun-Earth-probe (SEP) angles, thereby preventing outages due to the spacecraft line of sight (LOS) being in the proximity of the solar disk and the associated severe background contamination of the laser signal being transmitted from space. For example, for a spacecraft orbiting Mars, the outage during solar conjunction can be limited to 3 percent of a Martian year by designing a ground station that can operate while pointing within 3 degrees of the Sun. Moreover, the capability of predicting the atmospheric conditions, coupled with an effective handover strategy between the stations in the network (given the long light-times), is critical to achieving optimal operations with a ground network. In this article, the predictive models for weather and atmospheric conditions will not be addressed; instead, atmospheric channel statistics will be taken into account to determine some preliminary consideration about link availability and data volumes.

As elaborated in the following, the data needed to comprehensively address all of the above considerations are incomplete. However, some lower bounds can be predicted, based upon the limited data that are available and that are discussed below. Existing astronomical observatory sites share many of the attributes desired for deep-space optical receiving ground stations, with the additional requirement of operating not only in the daytime but at very small Sun-separation angles.

Finally, the data rates used to estimate data volumes utilize the Mars Laser Terminal (MLT) transmission characteristics, namely, a 5 -W average power laser transmitted by a 30 -cm-diameter diffractionlimited telescope. Furthermore, 5-m-diameter telescopes, similar to the Hale telescope at Palomar Mountain, are assumed for reception of the downlink at $1064 \mathrm{~nm}$. Single photon-counting detectors with 30 percent photo-detection efficiency are assumed for the optical receivers. In addition, 0.1-nm noise equivalent bandwidth (NEB) optical filters are used to reject out-of-band background light. The downlink is assumed to be circularly polarized so that an additional $3 \mathrm{~dB}$ of background discrimination can be achieved.

\section{Ground Network Configuration and Cloud Statistics}

Architectures and configurations for the Optical Deep Space Network (ODSN) have been conceptualized. $^{2}$ Some of the popular configurations described have been a linearly dispersed optical subnet (LDOS) and a clustered optical subnet (COS). As an example, these configurations are represented in Fig. 1 by stars (LDOS) and circles (COS), respectively.

The global distribution of the stations in the ODSN is configured to maintain LOS with planetary spacecraft as the Earth rotates. Furthermore, the ability to hand over between ground stations in the spacecraft LOS as a work-around against cloud blockage is intended. In order to achieve the highest network availability, adjacent nodes in either of the two configurations shown should be anti-correlated in cloud coverage.

\footnotetext{
${ }^{2}$ K. Shaik and M. Wilhelm, Ground Based Advanced Technology Study (GBATS); Optical Subnet Concepts for the DSN,
} Jet Propulsion Laboratory, D-11000, Release I, August 5, 1994. 


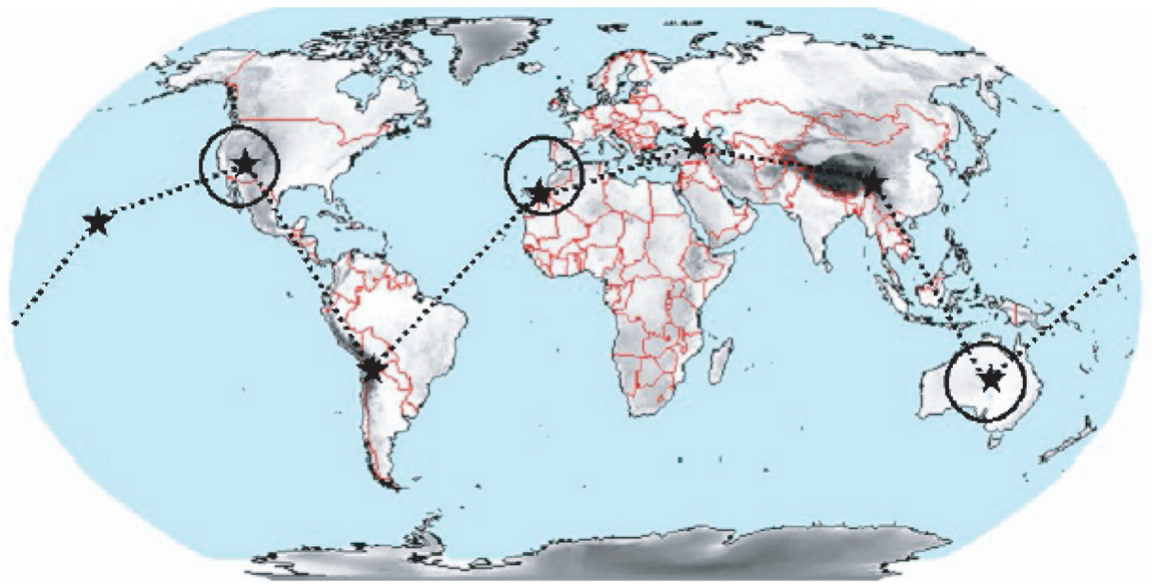

Fig. 1. Two alternate configurations for the ODSN: the LDOS (stars) and the $\cos$ (circles).

Studies based upon many years of National Climate Data Center surface observations [4] of cloud cover were analyzed for sites scattered over the Southwestern United States of America, and generally availability could be enhanced from 60 to 70 percent for a single site to 80 to 95 percent for a combination of three sites.

More recently, a study of cloud cover [5] was undertaken utilizing satellite imagery. The spatiotemporal resolution of this approach is determined by the pixel angular field of view and the frequency at which images are acquired. The spatial resolution is $5 \mathrm{~km}$, and the temporal resolution is 1 hour. CFLOS availability of sites predominantly in the Southwestern continental United States (CONUS) and Hawaii was determined. Various combinations of these sites then were analyzed to determine the overall CFLOS availability of a ground-based optical receiving network with a spacecraft assumed to be in Mars orbit. The details of this study are reported elsewhere [5]. Figure 2 summarizes the findings for a combination of a four-site cluster comprised of Goldstone, California; Kitt Peak, Arizona; McDonald Observatory, Texas; and Mauna Kea, Hawaii, where the CFLOS availability is presented by quarter. The overall CFLOS availability of this hypothetical network is 90 percent. The year 1998 stands out as an exception, most likely associated with El Niño. The relatively low first quarter availability is attributed to all the sites being in the Northern Hemisphere and therefore sharing a common winter season, as well as to the declination of Mars orbit causing the spacecraft not to rise very high in the sky and resulting in a relatively shorter pass. Therefore, reconfiguring the cluster to include a node in the Southern Hemisphere should enhance availability. Unfortunately, multi-year statistics cannot be verified for this last configuration because of the scarcity of data for a statistically significant duration. Based on one year's data, May 2003 to June 2004, it was found that a three-site cluster comprised of Kitt Peak (Arizona), Arequipa (Peru), and La Silla (Chile) provided a CFLOS of 98 percent, with the minimum availability being 96 percent during the month of January 2004 (Fig. 3).

To summarize, the cloud statistical study indicates that overall 90 percent availability can be achieved, based upon 6-years of data in the continental United States and Hawaii, and by extending the node of the network to South America, improved CFLOS statistics of 96 to 98 percent may be feasible, although further long-term data analysis is required to verify this.

\section{Performance During CFLOS}

Next the performance of a single node of a network during CFLOS is addressed. In this context, ground-station outages have to do with configuration of the station with respect to the planets and the Sun and the variability of atmospheric parameters. With regard to station configuration, as pointed out 


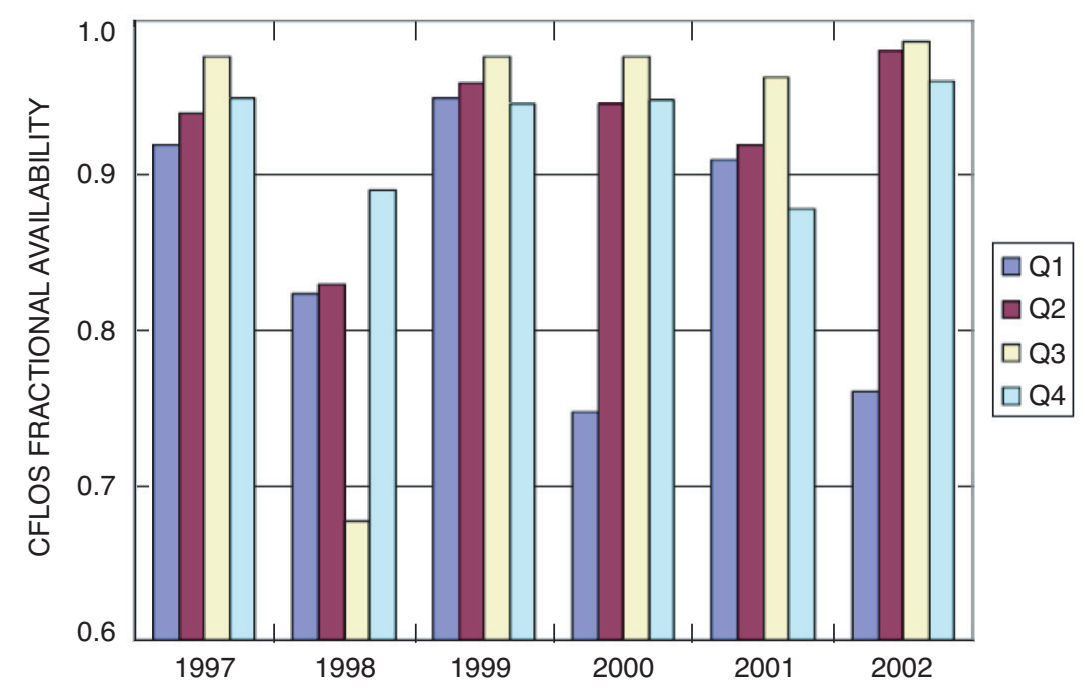

Fig. 2. Summary of CFLOS availability for a 4-site cluster comprised of Goldstone, California; Kitt Peak, Arizona; McDonald Observatory, Texas; and Mauna Kea, Hawaii, shown by quarter for 6 years.

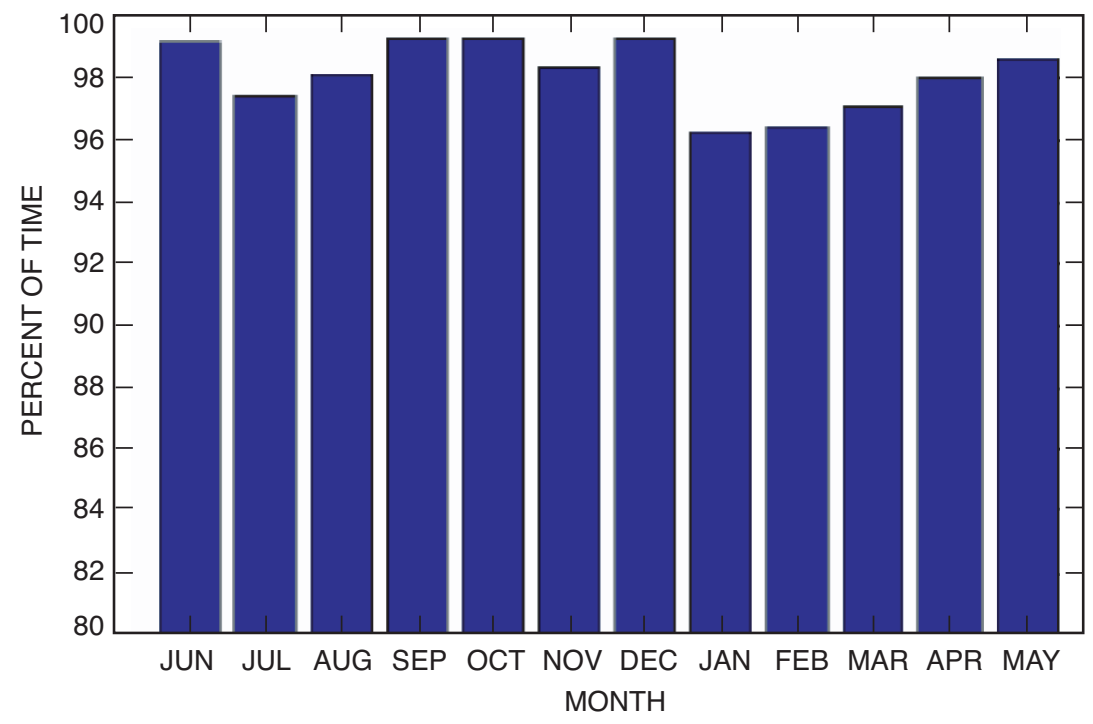

Fig. 3. Diversity CFLOS statistics for the period from May 2003 to June 2004 for the sites in Kitt Peak, Arizona; Las Silla, Chile; and Arequipa, Peru.

earlier, for a spacecraft orbiting Mars, an outage of 3 percent of the Martian year is introduced due to the inability of the space and ground terminals to "stare" at the Sun. An additional 2 percent outage is associated with eclipsing of the spacecraft by the planet Mars, although this is not unique to optical communications.

As for atmospheric variability attenuation, sky radiance and atmospheric "seeing" are the key parameters that display seasonal as well as diurnal variations. The MLCD Project will provide a good opportunity for evaluating the impact of atmospheric variability on link performance. The discussion that follows makes predictions based on limited available data and their statistical variations. 


\section{A. Atmospheric Attenuation}

Atmospheric modeling tools such as MODTRAN [6] can predict atmospheric transmittance and sky radiance for a variety of atmospheric and site-specific conditions. Figure 4 shows the loss due to atmospheric attenuation at wavelengths of 860 and $1064 \mathrm{~nm}$, in decibels, for three different atmospheric aerosol models, namely, desert extinction with $12-\mathrm{m} / \mathrm{s}$ wind speed; rural aerosol with clear sky, or a $23-\mathrm{km}$ visibility range; and rural aerosol with sub-visual cirrus, 5 - $\mathrm{km}$ visibility range. The loss is shown for zenith angles of 0 and $70 \mathrm{deg}$. The two wavelengths were chosen so that the model predictions could be compared to existing data, as described below. A ground station maximum viewing zenith angle of $70 \mathrm{deg}$ is assumed, starting from when the spacecraft rises above the ground receiving site. The attenuation will decrease as the spacecraft rises to its maximum zenith angle and then will start increasing again. Link performance of course is related to the atmospheric attenuation; however, knowledge of site-specific statistical fluctuations of attenuation are needed in order to derive the diurnal and seasonal variations in signal strength. In other words, how often which model should be used to predict attenuation at a particular site is not obvious, bearing in mind that the attenuation referred to here is during CFLOS, when optical links can be established and maintained.

Spectral attenuation of the atmosphere is being measured at Table Mountain, California, and Mount Lemmon (ML), Arizona, using starlight transmission with JPL Atmospheric Visibility Monitoring (AVM) [7] stations. Figure 5 shows a quarterly cumulative distribution function for atmospheric attenuation loss for a local zenith LOS at the Table Mountain Facility (TMF). The AVM stations use commercially available, silicon back-thinned charge-coupled devices (CCDs) for recording star images. The measured star signal is processed to determine atmospheric attenuation at wavelengths of $860 \mathrm{~nm}$ and $1064 \mathrm{~nm}$. The sensitivity at $1064 \mathrm{~nm}$ in the presence of daytime backgrounds is poor, and reliable statistics cannot be gathered. Therefore, the data at $1064 \mathrm{~nm}$ represents predominantly nighttime attenuation statistics, whereas the $860-\mathrm{nm}$ data are representative of both daytime and nighttime statistics. Table 1 shows spectral zenith attenuation in decibels as a function of percentage cumulative probabilities. The data are predominantly from Table Mountain, California, with Mount Lemmon data for 3 months. The blanks in Table 1 indicate that no data were acquired. With the simple assumption that an attenuation loss larger than $3 \mathrm{~dB}$ represents a link outage, the overall availability from the viewpoint of spectral attenuation will be 70 to 80 percent. This is consistent with the 6 -year cloud-cover study report [5], where 65 percent was

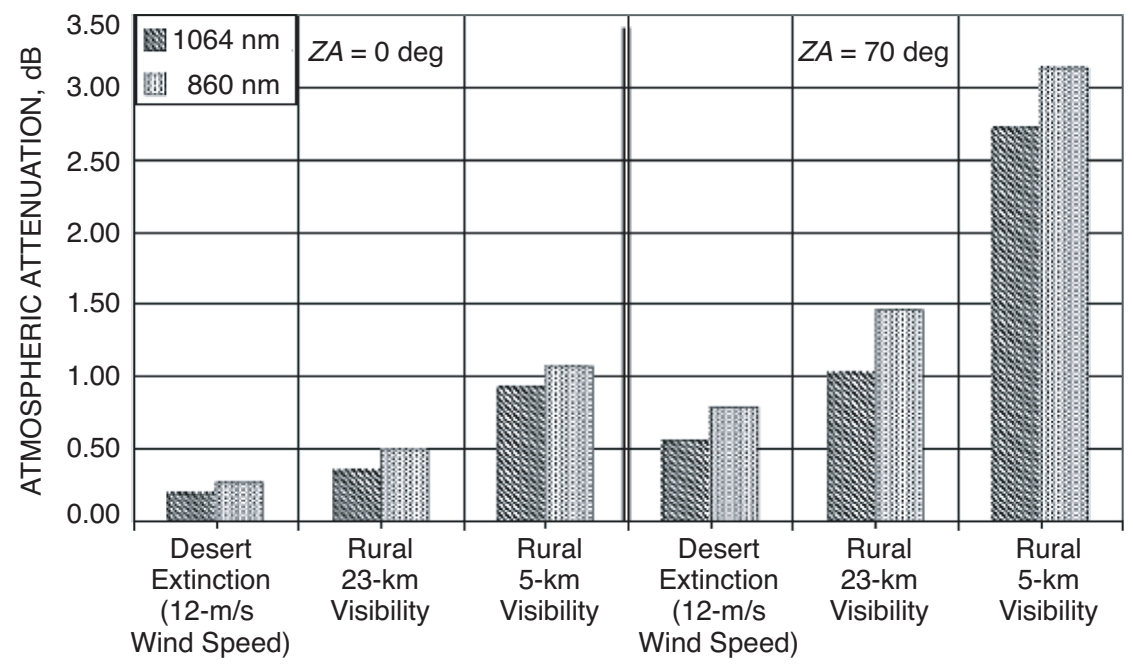

Fig. 4. Atmospheric attenuation at $860-$ and $1064-\mathrm{nm}$ predicted by MODTRAN for desert extinction with $12-\mathrm{m} / \mathrm{s}$ wind speed, rural aerosols with 23-km clear sky, and rural aerosol with hazy sky for two zenith angles of 0 and $70 \mathrm{deg}$. 


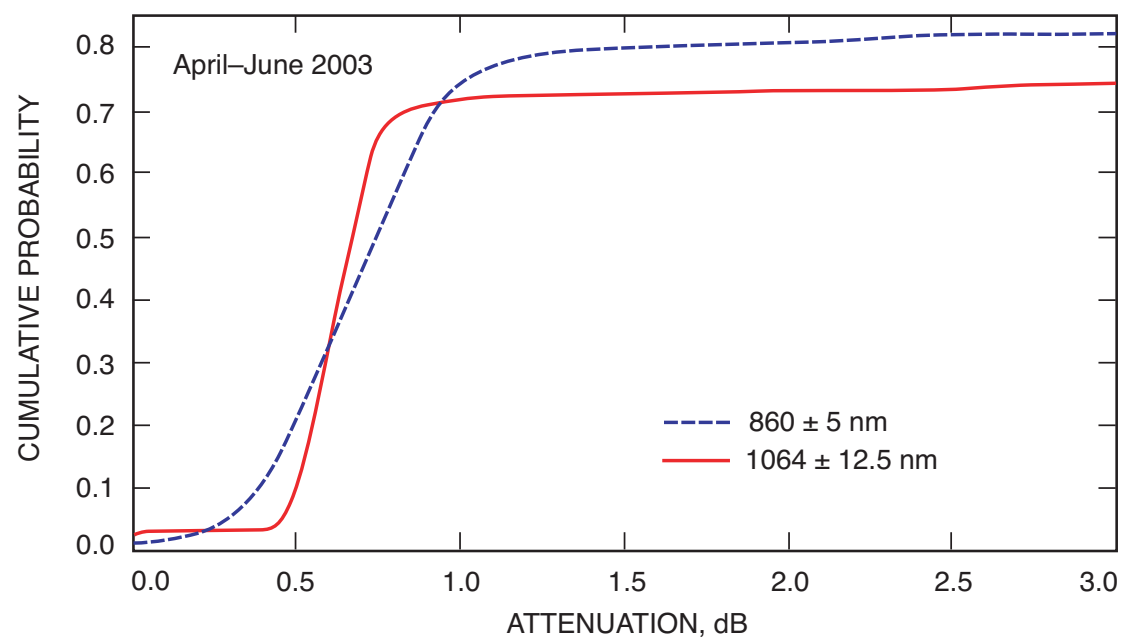

Fig. 5. Typical cumulative distribution functions for atmospheric attenuation measured with the AVM station at Table Mountain, California, during the second quarter of 2003.

Table 1. Summary of the percentage cumulative probability of measuring spectral attenuation at 1064 and $860 \mathrm{~nm}$ for 2002 and 2003 from Table Mountain, and one-quarter of the data from Mount Lemmon.

\begin{tabular}{llllll}
\hline Location & $\begin{array}{c}\text { Station } \\
\text { uptime } \\
\text { fraction }\end{array}$ & $<2 \mathrm{~dB}$ & $<1.5 \mathrm{~dB}$ & $<1 \mathrm{~dB}$ & $<0.5 \mathrm{~dB}$ \\
\hline
\end{tabular}

\begin{tabular}{|c|c|c|c|c|c|c|}
\hline \multicolumn{7}{|c|}{ 1064-nm with $25-\mathrm{nm}$ bandpass } \\
\hline $\mathrm{TMF}$ & Q3, 2002 & 0.33 & 0.8 & 0.79 & 0.73 & 0.15 \\
\hline TMF & Q4, 2002 & 0.62 & 0.62 & 0.6 & 0.56 & 0.45 \\
\hline TMF & Q1, 2003 & 0.85 & - & - & - & - \\
\hline ML & Q1, 2003 & 0.4 & 0.62 & 0.58 & 0.5 & 0.2 \\
\hline $\mathrm{TMF}$ & Q2, 2003 & 0.85 & 0.73 & 0.73 & 0.7 & 0.4 \\
\hline TMF & Q3, 2003 & 0.7 & 0.68 & 0.65 & 0.53 & 0.4 \\
\hline \multicolumn{7}{|c|}{$860-\mathrm{nm}$ with $10-\mathrm{nm}$ bandpass } \\
\hline TMF & Q3, 2002 & 0.33 & 0.87 & 0.83 & 0.78 & 0.35 \\
\hline TMF & Q4, 2002 & 0.62 & 0.65 & 0.62 & 0.58 & 0.35 \\
\hline TMF & Q1, 2003 & 0.85 & 0.4 & 0.35 & 0.25 & 0.05 \\
\hline ML & Q1, 2003 & 0.4 & 0.5 & 0.46 & 0.45 & 0.27 \\
\hline $\mathrm{TMF}$ & Q2, 2003 & 0.85 & 0.83 & 0.8 & 0.75 & 0.4 \\
\hline $\mathrm{TMF}$ & Q3, 2003 & 0.7 & 0.81 & 0.72 & 0.53 & 0.2 \\
\hline
\end{tabular}


reported for the single-site availability for Table Mountain, California. Moreover Table 1 shows that in the winter quarter the attenuation can reduce single-site availability to $40-50$ percent. For the winter quarter of 2003, Mount Lemmon shows an overall lower attenuation compared to Table Mountain at $860 \mathrm{~nm}$.

If Table Mountain were a node of a ground-based network, then other nodes would have to be relied upon to provide the overall 90 to 98 percent availability discussed earlier. For durations when links can be established, the zenith attenuation will vary from less than $0.5 \mathrm{~dB}$ (about 10 percent or less of the time) to 1 to $1.5 \mathrm{~dB}$ (70 to 80 percent of the time) with some exceptions when the upper availability percentages could dip down to 50 to 60 percent.

\section{B. Sky Radiance}

Two-thirds of the deep-space optical links from Mars occur during daytime. Therefore, sky radiance is a dominant source of additive background light for the link. Even though ultra-narrow optical bandpass filters, polarization discrimination, and spatial filtering will be used to reject the additive background contributed by sky radiance, it will remain a dominant source of background photons that are incident on the detector, especially at low SEP angles. Predicting the bounds of sky radiance can be achieved by the use of the same MODTRAN models mentioned in Section III.A. Once again site-specific measurements must be relied upon in order to gain insight into the statistical variation of the sky radiance.

Figure 6 compares sky radiance predicted by MODTRAN for a wavelength of $1064 \mathrm{~nm}$ and in the principal plane of the Sun (under assumptions identical to those used for attenuation in Fig. 4) with similar measurements made at Table Mountain, California, at a wavelength of $1020 \mathrm{~nm}$. The measurements were

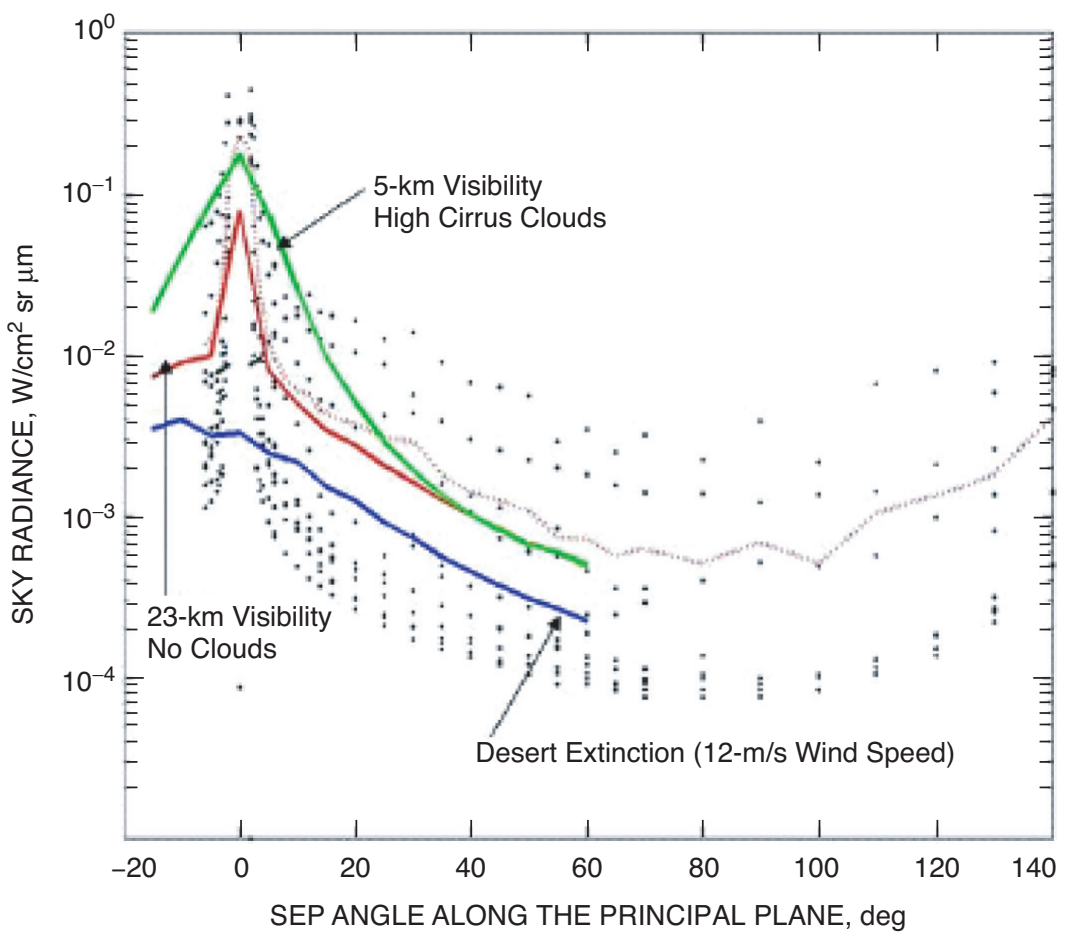

Fig. 6. Sky radiance predicted by MODTRAN (solid lines) superimposed on measurements made during January and February 2000 at Table Mountain, California. The dotted line represents the average of measured sky radiance measurements made at Sun zenith angles of 50 to $55 \mathrm{deg}$. 
made using a CIMEL Sun photometer used by the Aerosol Robotic Network (AERONET) [8]. The measurements represent variation of sky radiance with Sun separation angle for observations made at a Sun zenith angle of 50 to $55 \mathrm{deg}$. The peak shown on the theoretical predictions indicates a 0 -deg Sun separation and can be considered as a singularity. Figure 6 illustrates the good agreement between measurements and model predictions.

Figure 7 shows a cumulative distribution function of sky radiance measured at Table Mountain, California. The distributions represent data acquired during two campaigns during January and February 2000 and from June 2003 to January 2004. Measurements were made at Sun separation angles of 3 deg and combine data acquired along the principal plane and in almucantar. The dependence on the location of the Sun in the sky is also shown. The fact that on both occasions the sky radiance was larger at lower Sun zenith angles is not understood, because models would suggest higher values of sky radiance at larger zenith angles due to the greater air-mass and scattering volume. This anomaly was not observed from other AERONET sites investigated.

\section{Atmospheric Seeing}

Atmospheric "seeing" determines the spatial blurring of the spot at the focal plane $[9,10]$ of an optical receiver. In other words, the smallest solid angle of sky imaged in order to collect the laser signal is determined by prevalent atmospheric seeing that is related to the atmospheric coherence length (Fried parameter, $\left.r_{0}\right)$. Therefore, the ground receiving telescope field of view (FOV) is related to atmospheric seeing. Larger FOVs are required for smaller values of $r_{0}$. The larger FOV implies a larger portion of the sky being imaged on the detector, and hence it will result in an increase in additive background light, contributed predominantly from sky radiance, but also from non-negligible contributions from Mars when it is in the FOV and off-axis scattered light due to direct solar illumination of the ground receiving telescope aperture. In order to assess the combined effects of sky radiance and atmospheric seeing with the contributions from Mars and scattering factored in, joint probability statistics of sky brightness and atmospheric seeing for a given site are required. Desirable nodes for an optical receiving network should

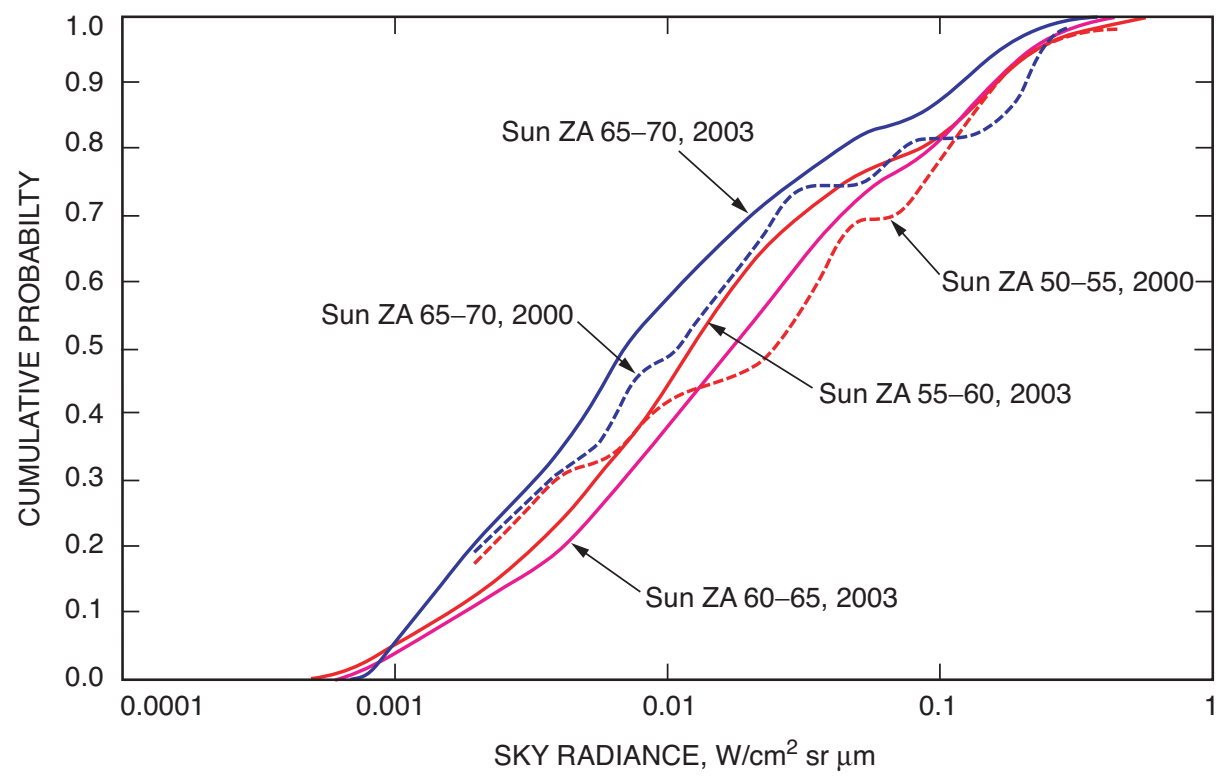

Fig. 7. Cumulative probability distribution of sky radiance measured at Table Mountain, California, during January and February 2000 and from June 2003 to January 2004. Measurements were made at a Sun separation of 3 deg both along the principal plane and in almucantar. 
provide favorable joint probability statistics. Current data on these two atmospheric parameters are not readily available from sites of interest to optical communications. The MLCD Project is planning to implement this capability by the beginning of the year 2007. In the meantime, for the purposes of our analysis, the sky radiance statistics are combined with worst-expected seeing in order to provide bounds on data volumes achievable.

\section{Availability and Data Volume}

The foregoing discussion should make it apparent that predicting data volumes for a global optical receiving network is a complex problem since statistically significant distributions of a number of sitespecific atmospheric parameters in addition to cloud-blockage statistics are needed. Addressing this is beyond the scope of the present article.

A simplified analysis intended to identify a lower bound of data volume, under worst-case link conditions (maximum range and minimum SEP angles of 3 to $10 \mathrm{deg}$ ) for a Mars-orbiting spacecraft, communicating to a ground-based network will be considered. In order to do so, we fix seeing to be $10 \mathrm{~cm}$ and $6.8 \mathrm{~cm}$ at 55-deg and 70-deg zenith angles. These zenith angles were chosen based on the assumption that the ground station cannot look beyond $70 \mathrm{deg}$ due to local topology and the tree-line, whereas the spacecraft does not rise over 55 deg during the solar conjunction of 2010-2011 (Mars Telecom Orbiter (MTO) Mission) for a Southwestern United States ground site. Utilizing the distribution of sky radiance and the fixed $r_{0}$, as well as accounting for the atmospheric attenuation distributions discussed, the average background light and signal photons are plotted as squares in Fig. 8. The background estimates include a fixed allocation of 0.02 photons/ns contributed by sunlight reflected from Mars and 0.03 photons/ns of stray light. For comparison, the diamonds and triangles show the increase in signal and reduction in background as range decreases and SEP angle increases.

Thus, over the SEP angle range from 3-10 deg (maximum Mars range of $2.4 \mathrm{AU}$ in 2011) to $160 \mathrm{deg}$ (minimum Mars range of 0.67 AU in 2012), nearly three decades of background and more than one decade of signal variation occur. Note that this combination of signal and background was obtained for the MLT parameters and a 5-m-diameter aperture telescope similar to the Hale Telescope on the ground. The laser

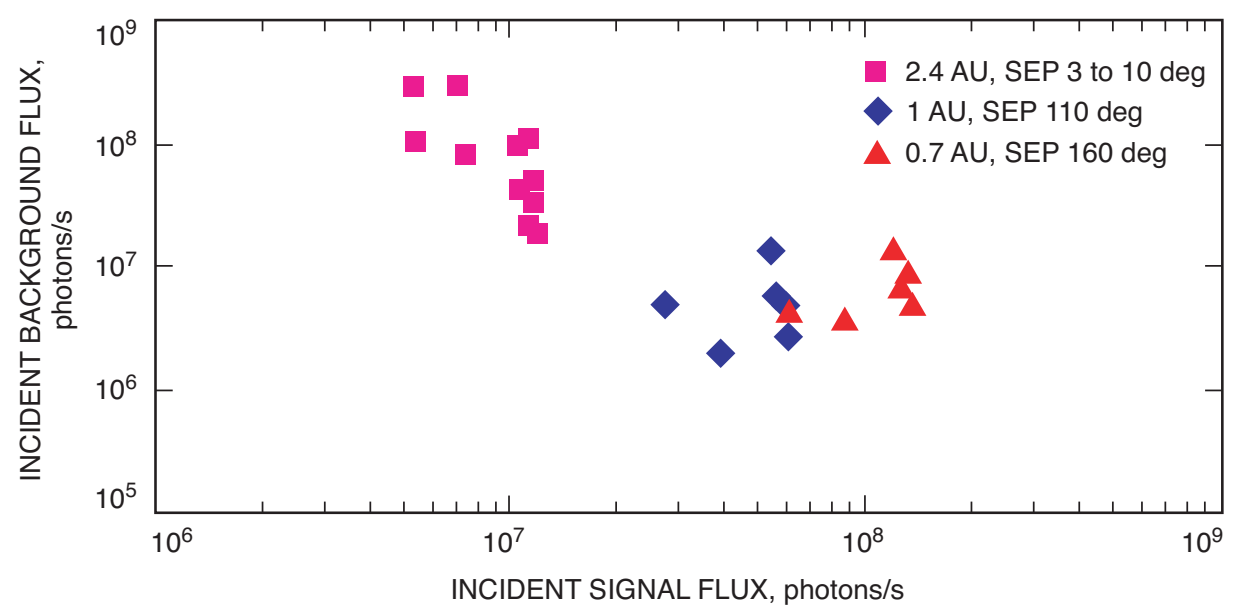

Fig. 8. Distribution of signal and background for optical downlink during a single Mars orbit. The statistical variation of sky radiance and atmospheric attenuation for fixed "seeing" was accounted for during maximum range (2.4 $\mathrm{AU}$ ) and 3 to $10 \mathrm{deg}$ SEP angle (diamonds). The other data at 1 and $0.7 \mathrm{AU}$ are nominal and included for reference. 
transmitter had nominally 5 -W average power and was limited to a peak power of $300 \mathrm{~W}$. Pulse-position modulation (PPM) is assumed with alphabet sizes of 32 and 64. Moreover, the statistical sky radiance and atmospheric attenuation are representative of values measured at Table Mountain, California.

The signal and background are incident upon a photon-counting detector with 30 percent detection efficiency and $10^{6}$ counts per second of dark noise. Included in the link losses are a 1-dB receiver implementation loss and a 1-dB decoder implementation loss. The latter includes the gap from theoretical capacity that the serially concatenated PPM code [11] can achieve.

Representative data rates for the signal and background photon fluxes plotted in Fig. 8, with a fixed code rate of 0.5 and a $3-\mathrm{dB}$ link margin, are shown in Fig. 9. The data rates displayed are constrained by the effective slot widths that the MLCD signaling scheme is planning to implement. They are plotted as cumulative percentages. Thus, for SEP angle between 3 and $10 \mathrm{deg}$, about 40 percent of the time mean data rates of $0.34 \mathrm{Mb} / \mathrm{s}$ will be achievable, whereas the remaining 60 percent of the time mean data rates of $1.4 \mathrm{Mb} / \mathrm{s}$ will be realized. Likewise, at SEP angles of $110 \mathrm{deg}$ at a $1 \mathrm{AU}$ range, 33 percent of the time a mean of $10 \mathrm{Mb} / \mathrm{s}$ will be achievable while 66 percent of the time $28 \mathrm{Mb} / \mathrm{s}$ will be realized. Likewise, at the closest range to Mars of $0.7 \mathrm{AU}, 17$ percent of the time mean data rates of $28 \mathrm{Mb} / \mathrm{s}$ will be realized, whereas 83 percent of the time $47 \mathrm{Mb} / \mathrm{s}$ will be achieved.

Based on the foregoing, a rather simple estimate of the lower bound of data volume can be made. For example, with the cluster of the Northern Hemisphere sites mentioned in Section II, namely, Goldstone, Kitt Peak, McDonald Observatory, and Hawaii, 90 percent of the time a link to Mars can be made. However, during the low SEP period, a typical pass duration will be approximately 6 hours in duration. Therefore, if this cluster formed a node of the global network, a conservative estimate of the data volume would be 19 to 20 Gbits/pass. If a cluster comprised of Southern Hemisphere sites, namely, Kitt Peak, Arequipa, and La Silla, were used instead, the effective pass duration would be longer, and the data volume would increase to $29 \mathrm{Gbits} /$ pass. Note that for a global network assuming two other similar clusters with comparable availability, when Mars is at the farthest range and the SEP angles are small, 3 to $10 \mathrm{deg}$, the data volumes per day would vary from 60 to $90 \mathrm{Gbits} /$ day. On the other hand, when Mars is close to Earth, the data volumes could be as high as $1.55 \mathrm{Tbits} /$ pass or $4.650 \mathrm{Tbits} /$ day. In the latter estimate, a distinction is not made between Northern and Southern Hemisphere sites; instead, a long pass duration of 11 hours per cluster is assumed. Furthermore, the data volume estimates mentioned

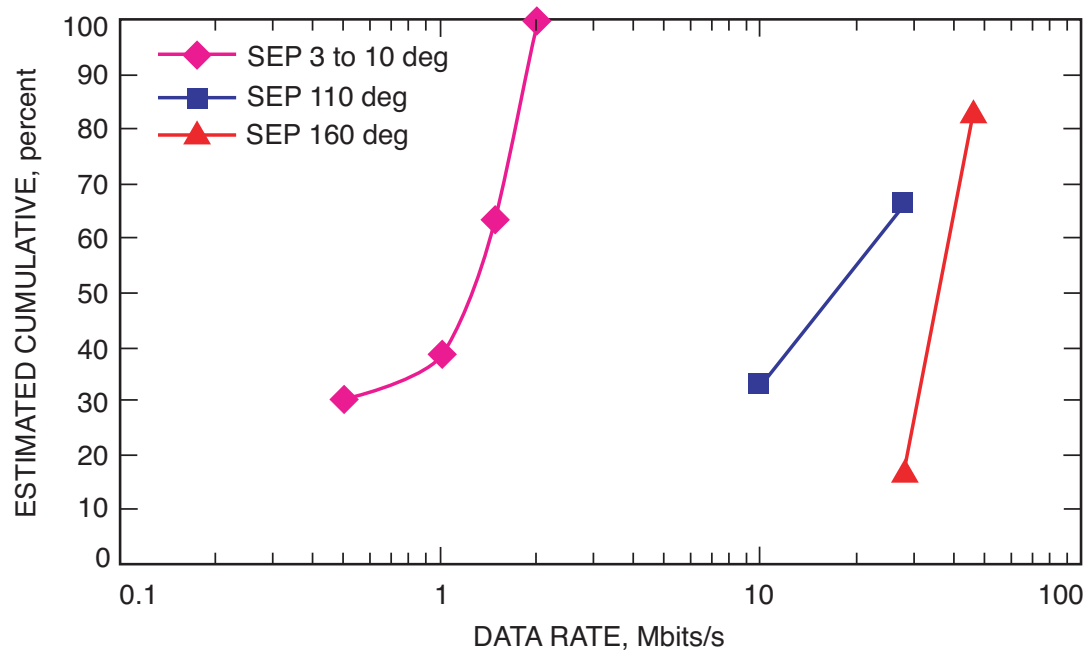

Fig. 9. Representative data rates corresponding to the signal and background light combinations shown in Fig. 8. 
here are within the constraints of the MLCD Project transmitter receiving aperture diameter $(5 \mathrm{~m})$ and signaling scheme with quantized slot widths. A 3-dB margin is assumed on all the data-rate estimates.

Note that the upper bound on the data volumes for the closest range to Mars is also conservative and restricted by what the MLCD Project is planning to implement. If this constraint were removed, the data volumes could be increased significantly.

\section{Conclusions}

The estimating of data volumes from an optical link is an interesting and complex problem, and the analysis presented above is a simplified and very preliminary estimate. The outages caused by the laserterminal-bearing spacecraft being eclipsed by Mars are not accounted for in the analysis. The planned upcoming Mars Laser Communications Demonstration (MLCD) will target measurements that will lend credibility to the basis upon which the estimates are made, namely, link availability and performance in the presence of atmospheric perturbations. An important conclusion from the above discussion is the need to configure ground receivers so that seasonal cloud coverage and orbital geometries do not extract too severe a penalty from achievable performance.

\section{Acknowledgments}

The authors would like to thank Mark Helmlinger for help in providing the AERONET sky radiance data, Ed Seykora for discussions on daytime seeing measurements, and Mitchell Troy for providing valuable insights on atmospheric seeing at Palomar Mountain.

\section{References}

[1] B. L. Edwards, S. A. Townes, R. S. Bondurant, J. J. Scozzafava, D. M. Boroson, W. T. Roberts, A. Biswas, A. D. Pillsbury, F. I. Khatri, J. W. Burnside, D. R. Bold, D. V. Murphy, A. K. McIntosh, D. O. Caplan, A. E. DeCew, J. Sharma, B. A. Parvin, R. J. Fitzgerald, S. H. Zingales, and R. DePaula, "Overview of the Mars Laser Communications Demonstration Project," Paper 2003-6417, AIAA Space 2003 Conference, Long Beach, California, September 23-25, 2003.

[2] D. Boroson, A. Biswas, and B. L. Edwards, "MLCD: Overview of NASA's Mars Laser Communications Demonstration System," SPIE Proceedings, vol. 5338, Free Space Laser Communications Technologies XVI, ed., S. Mecherle, January 27, 2004.

[3] D. Boroson, R. S. Bondurant and J. J. Scozzafava, "Overview of High Rate Deep Space Laser Communications Options," SPIE Proceedings, vol. 5338, Free Space Laser Communications Technologies XVI, ed., S. Mecherle, January 27, 2004.

[4] S. Piazzolla and S. Slobin, "Statistics of Link Blockage Due to Cloud Cover for Free-Space Optical Communication Using NCDC Surface Weather Observation Data," SPIE Proceedings, vol. 4635, Free Space Laser Communications Technologies XIV, ed., S. Mecherle, pp. 138-149, 2002. 
[5] M. E. Craddock, R. J. Allis, R. P. Link, S. Applegate, K. G. Davison, and K. E. Wilson, "Mitigating the Effects of Clouds On Optical Communications," SPIE Proceedings, vol. 5338B, Free-Space Laser Communication Technologies XVI, ed., S. Mecherle, January 29, 2004.

[6] A. Berk, L. S. Bernstein, and D. C. Robertson, MODTRAN: A Moderate Resolution Model for LOWTRAN 7, Air Force Geophysics Laboratory Technical Report GL-TR-89-0122, Hanscom AFB, Massachusetts.

[7] B. Sanii, A. Datta, D. Tsiang, J. Wu, and A. Biswas, "Preliminary Results of an Upgraded Atmospheric Visibility Monitoring Station," The Telecommunications and Mission Operations Progress Report 42-142, April-June 2000, Jet Propulsion Laboratory, Pasadena, California, pp. 1-12, 2000.

http://tmo.jpl.nasa.gov/tmo/progress_report/42-142/142K.pdf

[8] http://aeronet.gsfc.nasa.gov

[9] F. I. Khatri, D. M. Boroson, D. V. Murphy, and J. Sharma, "Link Analysis of Mars-Earth Optical Communications System," SPIE Proceedings, vol. 5338A, Free Space Laser Communications Technologies XVI, ed., S. Mecherle, January $27,2004$.

[10] A. Biswas and S. Piazzolla, "Deep-Space Optical Communications Downlink Budget from Mars: System Parameters," The Interplanetary Network Progress Report 42-154, April-June 2003, Jet Propulsion Laboratory, Pasadena, California, pp. 1-38, August 15, 2003.

http://ipnpr.jpl.nasa.gov/progress_report/42-154/154L.pdf

[11] B. Moision and J. Hamkins, "Deep-Space Optical Communications Downlink Budget: Modulation and Coding," The Interplanetary Network Progress Report 42-154, April-June 2003, Jet Propulsion Laboratory, Pasadena, California, pp. 1-28, August 15, 2003.

http://ipnpr.jpl.nasa.gov/progress_report/42-154/154K.pdf 